\title{
Stent Retriever Use for Retrieval of Displaced Microcoils: A Consecutive Case Series
}

\author{
T.M. Leslie-Mazwi, M. Heddier, H. Nordmeyer, M. Stauder, A. Velasco, P.J. Mosimann, and R. Chapot
}

\begin{abstract}
SUMMARY: Coil displacement during endovascular coiling procedures may require coil retrieval in the context of flow limitation or thromboembolic risk. No standard recommended method of coil retrieval exists. We present a consecutive series of 14 patients with displaced coil during aneurysm coiling in whom the complication was effectively managed with the use of a stent retriever system. Two illustrative cases from the 14 are described, and technical notes are detailed regarding use of the technique. The use of stent retrievers presents a simple, safe, and effective choice for removal of prolapsed coils during aneurysm coiling.
\end{abstract}

ABBREVIATION: $A C A=$ anterior cerebral artery

$\mathbf{E}^{\mathrm{n}}$ ndovascular occlusion of intracranial aneurysms is now widely practiced, with positive results in direct comparisons to open surgery. ${ }^{1}$ However, the occurrence of coil displacement and migration carries the risk for thromboembolic complications ${ }^{2}$ Therefore, if present during a case, coil displacement often requires either retrieval or fixation of the errant coil.

Stent retrievers have been introduced recently for the treatment of ischemic stroke, enabling rapid and reliable extraction of thrombus. ${ }^{3}$ We present a consecutive series of 14 cases in which off-label application of a stent retriever allowed retrieval of displaced intracranial aneurysm coils.

\section{MATERIALS AND METHODS}

We reviewed our prospectively collected data base of combined symptomatic and incidental aneurysm coiling cases, from January 2009 to August 2012. A total of 1125 consecutive aneurysms were treated during this time in our institution. Technique was similar for all, involving dual antiplatelet therapy in unruptured aneurysms, anticoagulation with heparin, use of MicroVention, (Aliso Viejo, California) coils, and systematic balloon assistance when necessary. Thirteen consecutive cases were prospectively identi-

Received October 18, 2012; accepted after revision January 6, 2013.

From the Department of Interventional Neuroradiology (T.M.L.-M., M.H., H.N., M.S., A.V., P.J.M., R.C.) Alfried-Krupp-Krankenhaus, Essen, Germany; Department of Interventional Neuroradiology/Endovascular Neurosurgery (T.M.L.-M.), Massachusetts General Hospital, Boston, Massachusetts; and Department of Radiology and Neuroradiology (P.J.M.), Centre Hospitalier Universitaire Vaudois and University of Lausanne, Lausanne, Switzerland.

Please address correspondence to R. Chapot, MD, Department of Intracranial Endovascular Therapy, Alfried-Krupp-Str. 21, 45131 Essen, Germany; e-mail:

rene.chapot@krupp-krankenhaus.de

http://dx.doi.org/10.3174/ajnr.A3552 fied in which intraprocedural coil displacement or migration occurred that required intervention. In all cases, the intervention chosen was application of a stent retriever, either the Solitaire retrievable stent (Covidien, Dublin, Ireland), or the Catch Plus device (Balt Extrusion, Montmorency, France).

\section{Patients}

A total of 14 patients (12/14 women), ranging in age from 29-73 years, met inclusion criteria. Aneurysms were of various sizes (4-28 mm) in various locations (ICA, MCA M1, anterior cerebral artery $[\mathrm{ACA}] \mathrm{A} 2$, and basilar artery). In every case, retrieval was successful, without a change in the stability of the aneurysm or in the patient's baseline neurologic examination. Six cases had retrieval with a single pass. One patient required a maximum of 7 passes, with a median of 2 and mean of 2.3 passes per patient.

A brief description of 2 illustrative cases follows.

Case 1. A 2-3 HyperSoft coil (MicroVention) dislodged and migrated into the pericallosal artery during placement at the neck in a patient with a ruptured anterior communicating artery aneurysm (Fig 1). A Rebar 18 microcatheter (ev3, Irvine, California) was navigated beyond the coil to enable a stent retriever (Solitaire $4 \times 20$, Covidien) to be delivered at the level of the coil. After partial resheathing of the stent retriever, the coil was trapped and the system progressively withdrawn, enabling retrieval of the coil.

Case 2. A single loop of coil prolapsed into the main trunk of the MCA after coiling of an unruptured MCA aneurysm. Despite effective dual antiplatelet therapy, a TIA occurred 2 days later related to clot formation on, and subsequent embolization from, the prolapsed coil loop. A decision was made to attempt coil removal. A stent retriever (Solitaire $4 \times 15$, Covidien) was delivered 

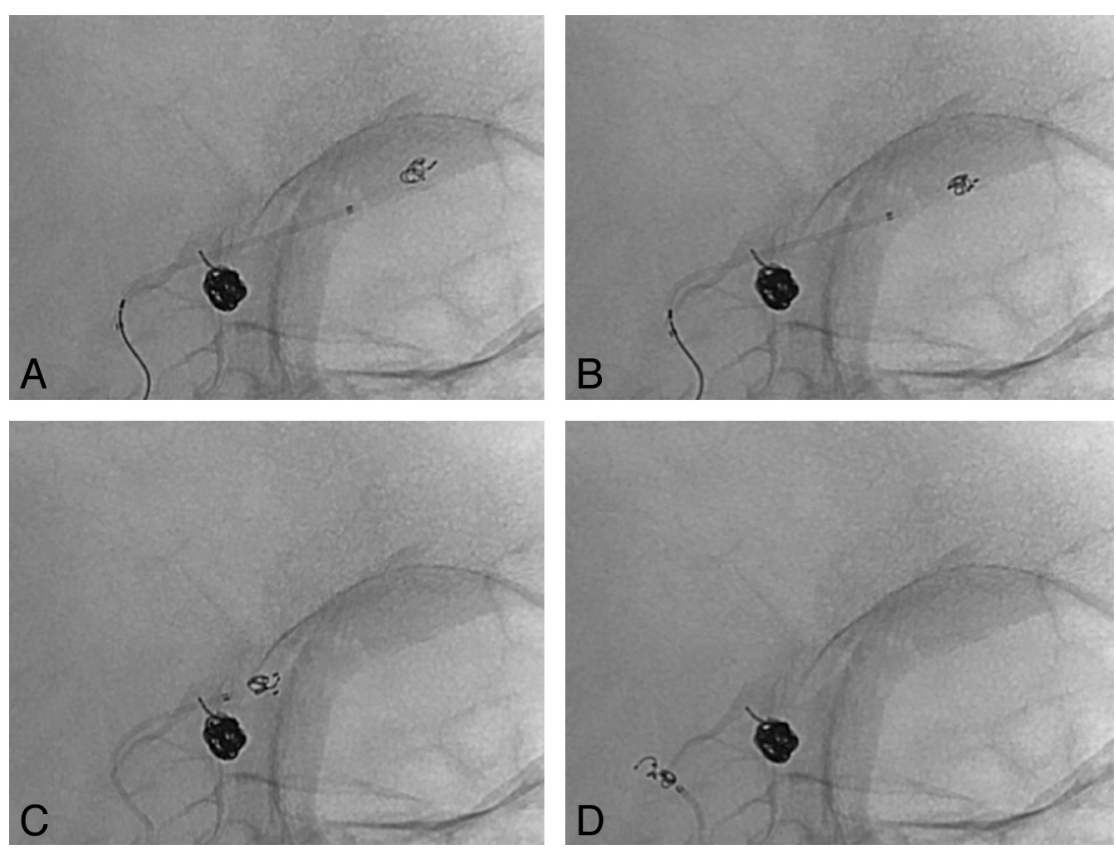

FIG 1. Coil retrieval. A, Displaced coil is visible in the more distal ACA, distinct from the coil mass within the anterior communicating artery aneurysm. The coiling microcatheter has been removed. The stent retriever delivery catheter is seen traversing the aneurysm neck, and the stent retriever has been deployed adjacent to the errant coil. $B$, Retraction of the stent shows capture of the displaced coil. Note the conformational change to the displaced coil mass. $C$, The stent retriever is pulled from the intracranial circulation with coil engaged, past the existing coil mass. $D$, The coil is successfully removed, without disruption of the remaining coil within the aneurysm. Additional coils were subsequently placed in the aneurysm to complete the procedure.

in the region of the coil by use of the technique described below, and gently withdrawn while only partially resheathed. The coil was then retrieved without dislocation of the other coils (Fig 2). The patient's symptoms resolved.

\section{DISCUSSION}

Aneurysm coiling seals the aneurysm from blood flow by inducing thrombosis within the lumen of the aneurysm. ${ }^{4}$ Thromboembolic complications remain the greatest risk in treatment of an endovascular aneurysm, with displaced coil material contributing significantly to this risk. ${ }^{2}$ In addition, the coil may migrate with blood flow into smaller-branch vessels or lodge at a vessel bifurcation, producing flow limitation. The risk for coil displacement and migration is influenced by a combination of anatomic and technical factors. Both undersized and/or unstable long coils can result in distal coil migration, especially in wide-neck aneurysms. ${ }^{5}$ Use of balloon or stent assistance minimizes the risks for coil displacement and migration; careful coil delivery to avoid prolapse of the catheter tip, and deployment of new coils within a stable coil basket additionally aim to minimize the risk for coil displacement.

In the absence of flow limitation or thrombus accumulation, many single loops of herniated coil are best left alone, and short infusions of intravenous glycoproteinIIb/IIIa antagonists provide protection from potential thrombus formation until fibrin coating makes the coil loops less thrombogenic. However, if displaced coil does require intervention, available options vary: broadly divisible into either stent placement to pin the coil down or active retrieval of the displaced coil loops. Fixation with a stent is an efficient technique ${ }^{6}$ that potentially avoids displacement of other coils during a retraction maneuver. Stent placement additionally requires less manipulation within the lumen of the affected vessel. However, as a salvage technique, it is often less attractive because of the concern of placing another thrombogenic intravascular implant, the need for dual antiplatelet therapy, and the possibility of multiple stents being required. The placement of a stent may also limit the ability to continue the definitive endovascular procedure. These concerns become particularly significant in the context of a ruptured aneurysm.

Hence, attempts at coil retrieval are often the primary strategy. Various methods to retrieve the loosened coil have been described. These include the use of microsnares, ${ }^{7,8}$ the Alligator retrieval device (Covidien), ${ }^{9}$ use of the Merci retriever (Concentric Medical, Mountain Veiw, California), ${ }^{10} \mathrm{mi}-$ crowire manipulation, ${ }^{11}$ and even microsurgery in the event of acute parent artery occlusion without the possibility of endovascular coil retrieval. ${ }^{12}$ The use of a stent for this role was first reported by Wakhloo and Gounis, ${ }^{13}$ who used a retrievable closed-cell intracranial stent to successfully extract coil material in an in vivo aneurysm model. Our initial experience with this technique was an attempt to fix an errant coil with a Solitaire AB stent. During this maneuver, we noted that the coil was engaged in the stent during stent movement, and therefore proceeded to retract it until it was cleared from the circulation. This was previously published by our group. ${ }^{14}$ We have since used the technique with success in a variety of scenarios. Use of a stent retriever in this role, as with many of the other techniques proposed, represents an off-label indication for the device.

We would make the following technical observations regarding use of a stent retriever device for coil retrieval:

1. Always decide whether the herniated coil requires intervention. Single loops of coil are usually best left undisturbed. Longer or unstable coil loops require definitive therapy. It is clear that any evidence of embolic phenomena or thrombus aggregation related to the coil requires intervention.

2. On identification of the complication, the patient should receive a loading dose of antiplatelet medication (preferably IV glycoprotein IIb/IIIa antagonists) if not already receiving these agents, and a heparin bolus should be administered if no strong contraindication exists. In the context of flow limitation, collateral augmentation should be pursued through fluid bolus and induced hypertension, if tolerated.

3. We have used stent retrievers comfortably in more proximal vessels, with coil retrieval from vessels the diameter of M2 MCA and A2 ACA vessels. In vessels $<2 \mathrm{~mm}$, the combination 

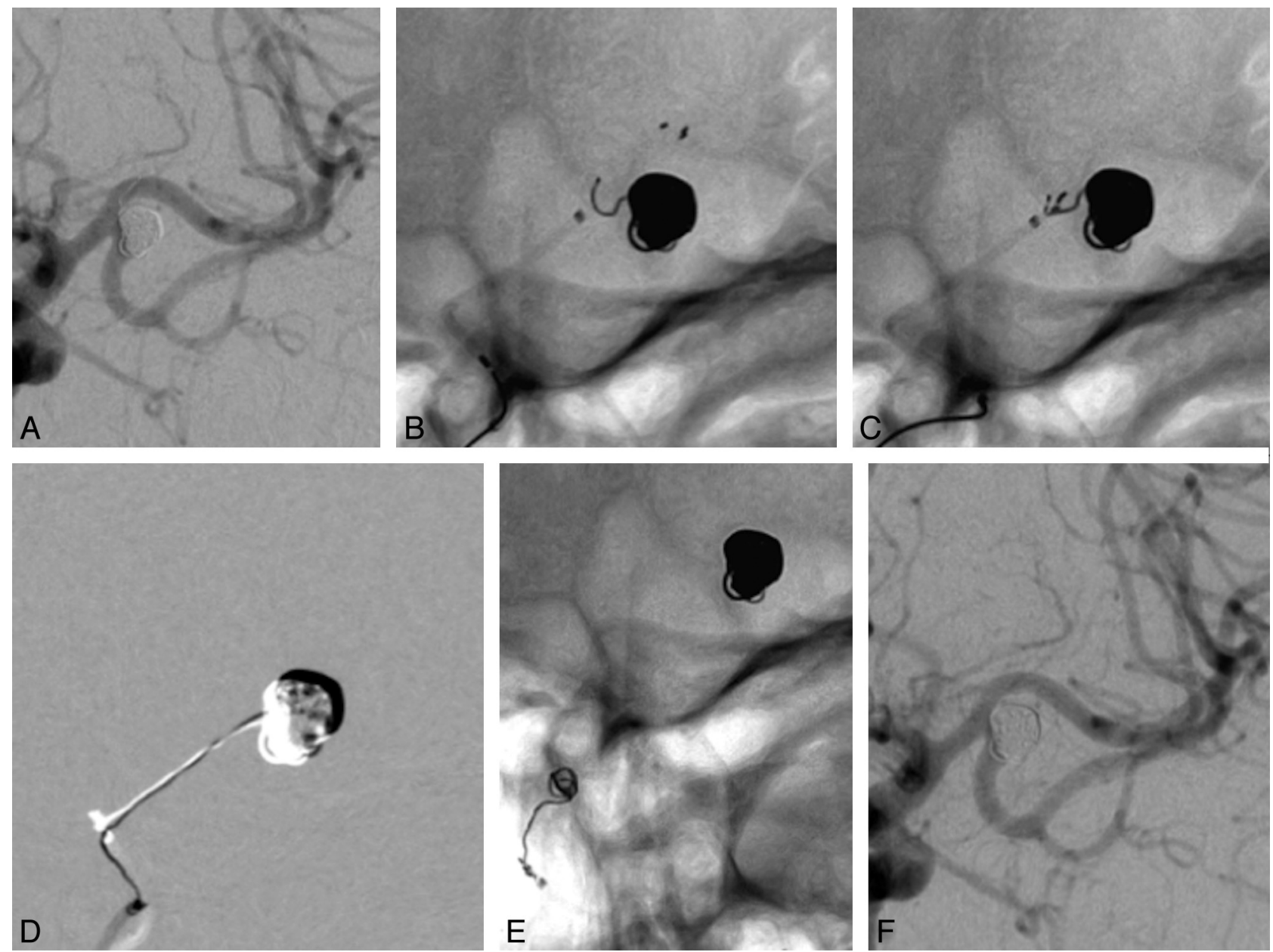

FIG 2. Delayed coil retrieval. A, Final coiling result, with coil loop protruding into the postbifurcation M1 segment of the MCA. The initial intention was to manage this conservatively. However, the patient had subsequent embolic events and required definitive treatment of the prolapsed coil. B, Deployment of the stent retriever, starting immediately distal to the prolapsed coil. C, Engagement of the coil, as evidenced by the conformational change in the coil loop appearance. $D$, Subtracted image showing traction on the coil loop during extraction from the aneurysm lumen. $E$, Successful removal of the offending coil from the circulation. $F$, Final result, with the aneurysm secured and unchanged in morphologic features after the removal of the prolapsed coil. The ischemic events in this patient did not recur.

of a small microcatheter (eg, 0.017 inner diameter) with dedicated distal access stent retrievers (eg, the Capture stent [MindFrame, Irvine, California] or the Catch Mini [Balt Extrusion]) may be preferred.

4. The intention should be unsheathing of the stent retriever immediately distal to the displaced coil (in our experience, within $5 \mathrm{~mm}$ of the distal coil). The stent retriever delivery catheter should be carefully navigated over a microwire until the tip is in the desired location for stent exposure. During this maneuver, minor movement of the displaced coil, including possible further distal migration, is not uncommon and should be expected. The ability to navigate the microcatheter distal to the coil may be a limiting factor, particularly when a significant coil mass is displaced and is potentially obstructive.

5. Delivery of the stent retriever is similar to the technique for thrombectomy in stroke. The stent retriever is partially unsheathed in the region of coil displacement, with just enough stent retriever exposed to completely cover the coil. This should be factored into catheter positioning before deployment. Coil-stent interaction should be maximized by slight forward pressure on the stent pusher wire to increase the potential for optimal stent deployment.

6. An attempt should then be made to resheath the stent retriever by advancing the microcatheter back over the device. Typically, this maneuver will trap the coil within the interstices of the stent retriever and pull the coil loops into the microcatheter with the stent retriever. Constant tension must be applied to the stent retriever to maintain the resheathed position, minimizing the likelihood of the coil working loose by securing it tightly in the retracted device.

7. Once the coil is successfully engaged, the system should be slowly withdrawn into the guide catheter with attention to potential movement of the rest of the coil mass. We had no patient in whom the coil mass within the aneurysm was displaced during this process. Anticipate that the coil will be extracted from the aneurysm intact.

8. Examine the coil retained within the stent retriever on removal. If the aneurysm and patient have remained stable, proceed with further coiling as indicated to complete the case.

9. Caution should be exercised in the context of previous place- 
ment of a stent, which may not prevent coils prolapse but inhibits the ability to safely remove coils with this method, as pulling a stent retriever through a pre-existing stent carries the risk for stent dislodgement. The main technical limitation is placement of the catheter tip distal to the coil, which may be especially challenging in smaller distal vessels $(<2$-mm diameter).

Previously, our preferred device for this clinical scenario was a microsnare, with 15 cases in the 13 years before January 2009 in which the snare-and-lasso technique was used. We were successful in 13 cases but failed to retrieve coils in 2 of the cases, and found the technical aspects significantly more challenging, with more vasospasm encountered and more attempts required to trap the coil. We also have experience with the Alligator retrieval device and have found that the progressive straightening of the teeth of the device limits the number of attempts that can be performed with each device (in our experience, no more than 2 or 3 attempts). In addition, the relative stiffness of the distal tip reduces the ability to orient the device well in a curved segment. Our experience with stent retrievers has proved much more consistent and reliable. Our current report includes consecutive patients, and it is noteworthy that the stent retriever device was successful in every case, with less technical manipulation than is required in many of the other techniques to retrieve stray coil. Despite prospective and consecutive collection of cases, it is important to note that our report had the limitations inherent in any limited, singlecenter case series. Although our findings relate to 2 specific stent retrievers, it seems reasonable, based on similar existing technology, that the principles described here would apply to any of the stent retrievers currently on the market. We cannot, however, comment on other systems with the data available at this time.

\section{CONCLUSIONS}

Stent retrievers are our first option in cases of coil displacement, presenting a simple, safe, and effective alternative for removal of a prolapsed coil during aneurysm coiling.

Disclosures: René Chapot-UNRELATED: Consultancy: ev3/Covidien,* MicroVention; ${ }^{*}$ Payment for Lectures (including service on speaker bureaus): ev3, ${ }^{*}$ MicroVention, ${ }^{*}$ Balt.* *Money paid to institution.

\section{REFERENCES}

1. Molyneux A, Kerr R; International Subarachnoid Aneurysm Trial (ISAT) Collaborative Group, et al. International Subarachnoid Aneurysm Trial (ISAT) of neurosurgical clipping versus endovascular coiling in 2143 patients with ruptured intracranial aneurysms: a randomized trial. J Stroke Cerebrovasc Dis 2002;11:304-14

2. Derdeyn CP, Cross DT 3rd, Moran CJ, et al. Postprocedure ischemic events after treatment of intracranial aneurysms with Guglielmi detachable coils. J Neurosurg 2002;96:837-43

3. Pereira VM, Narata AP, Gonzalez AM, et al. Use of stentrievers in acute stroke: tips, tricks, and current results. Tech Vasc Interv Radiol 2012;15:68-77

4. Bavinzski G, Talazoglu V, Killer M, et al. Gross and microscopic histopathological findings in aneurysms of the human brain treated with Guglielmi detachable coils. $J$ Neurosurg 1999;91:284-93

5. Eddleman CS, Welch BG, Vance AZ, et al. Endovascular coils: properties, technical complications and salvage techniques. J Neurointerv Surg 2013;5:104-09.

6. Schutz A, Solymosi L, Vince GH, et al. Proximal stent fixation of fractured coils: technical note. Neuroradiology 2005;47:874-78

7. Koseoglu K, Parildar M, Oran I, et al. Retrieval of intravascular foreign bodies with goose neck snare. Eur J Radiol 2004;49:281-85

8. Fiorella, D, Albuquerque FC, Deshmukh VR, et al. Monorail snare technique for the recovery of stretched platinum coils: technical case report. Neurosurgery 2005;57(1 Suppl): E210; discussion E210

9. Henkes $H$, Lowens S, Preiss $H$, et al. A new device for endovascular coil retrieval from intracranial vessels: Alligator retrieval device. AJNR Am J Neuroradiol 2006;27:327-29

10. Kung DK, Abel TJ, Madhavan KH, et al. Treatment of endovascular coil and stent migration using the Merci retriever: report of three cases. Case Report Med 2012;2012:242101

11. Lee CY. Use of wire as a snare for endovascular retrieval of displaced or stretched coils: rescue from a technical complication. Neuroradiology 2011;53:31-35

12. Kim YB, Lee KC, Lee JW, et al. Rescue microsurgery in coil herniation causing thromboembolic occlusion of parent artery. Acta Neurochir (Wien) 2009;151:1609-16

13. Wakhloo AK, Gounis MJ. Retrievable closed cell intracranial stent for foreign body and clot removal. Neurosurgery 2008;62(5 Suppl 2):ONS390-93; discussion ONS393-94

14. O'Hare AM, Rogopoulos AM, Stracke PC, et al. Retrieval of displaced coil using a Solitaire((R)) stent. Clin Neuroradiol 2010;20: 251-54 